\title{
ВОЗМОЖНОСТИ ИНТРАОПЕРАЦИОННОЙ ФЛУОРЕСЦЕНТНОЙ ДИАГНОСТИКИ С 5-АМИНОЛЕВУЛИНОВОЙ КИСЛОТОЙ В ХИРУРГИЧЕСКОМ ЛЕЧЕНИИ ГИПЕРПАРАТИРЕОЗА
}

\author{
Вшивцев Д.О., Ромащенко П.Н., Майстренко Н.А., Криволапов Д.С. \\ ФГБВОУ ВО «Военно-медицинская академия имени С.М. Кирова» МО РФ
}

Единственным радикальным методом лечения первичного (ПГПТ) и третичного гиперпаратиреоза (ТГПТ) является паратиреоидэктомия, успешность которой определяется полноценным удалением всех патологически измененных околощитовидных желез (ОЩЖ). На сегодняшний день продолжают разрабатываться и внедряться в клиническую практику интраоперационные методики флуоресцентной диагностики и поиска ОЩЖ, одной из которых является паратиреомониторинг с 5-аминолевулиновой кислотой (5-АЛК).

ЦЕЛЬ: определить возможности интраоперационной флуоресцентной диагностики околощитовидных желез с 5-амилевулиновой кислотой в хирургическом лечении первичного и третичного гиперпаратиреоза.

МАТЕРИАЛЫ И МЕТОДЫ: проанализированы результаты диагностики и хирургического лечения 9 больных ПГПТ и 1 ТГПТ. Предоперационное обследование больных и их послеоперационное ведение осуществляли в рамках существующих клинических рекомендаций. С целью топической диагностики ОЩЖ (паратиром) использовали сочетание не менее двух визуализирующих методик - ультразвуковое исследование с динамической сцинтиграфией ОЩЖ с 99mТс-технетрилом, ОФЭКТ, ОФЭКТ/КТ, МРТ шеи. За полтора часа до начала операции пациенты принимали раствор 5-АЛК в дозе 30 мг на 1 кг массы тела. Оперативные вмешательства выполняли с использованием минимально инвазивной эндоскопически-ассистированной методики (Minimally Invasive Video-Assisted Parathyroidectomy). Интраоперационный поиск ОЩЖ осуществляли с использованием источника поляризованного синего цвета (фонарик) с длиной волны 385-440 нм, направленного в рану в условиях затемненной операционной. При анализе полученных данных оценивали интенсивность флуоресценции здоровых и пораженных ОЩЖ, а также окружающих тканей, влияние 5-АЛК на течение послеоперационного периода.

РЕЗУЛЬтАТЫ: Селективная паратиреоидэктомия выполнена у 7 больных, билатеральная паратиреоидэктомия (удаление двух контралатеральных ОЩЖ) - у 2, тотальная паратиреоидэктомия (удаление всех ОЩЖ) - у 1. В ходе операции флуоресценция ОЩЖ (участки розового цвета) была получена у 8 пациентов. Интенсивность флуоресценции паратиром оказалась субъективно значительно выше, чем неизмененных ОЩЖ. Флуоресценции окружающих тканей и органов получено не было. Послеоперационные осложнения развились у 2 больных и были представлены фототоксической реакцией, связанной с фотосенсибилизирующими свойствами 5-АЛК $(\mathrm{n}=1)$, и транзиторной гипокальциемией, обусловленной объемом оперативного вмешательства при ТГПТ (n=1). Парезов гортани, персистенции и рецидива заболевания во всех случаях не отмечено.

\section{Выводы:}

1. Интраоперационная флуоресцентная диагностика ОЩЖ с 5-АЛК является безопасной вспомогательной методикой, которая позволяет осуществлять поиск и дифференциальную диагностику неизмененных ОЩЖ и паратиром, тем самым способствуя радикальности вмешательств, профилактике послеоперационного гипопаратиреоза, персистенции и рецидива заболевания.

2. Применение паратиреомониторинга целесообразно у больных с неоднозначными данными дооперационной топической диагностики ОЩЖ, при их множественном поражении, эктопированном расположении паратиром, при повторных операциях на щитовидной и околощитовидных железах, в том числе по поводу персистенции и рецидива гиперпаратиреоза.

КЛЮЧЕВЫЕ СЛОВА: флуоресчентная диагностика; первичный гиперпаратиреоз; третичный гиперпаратиреоз; Паратиреоидэктомия; 5-аминолевулиновая кислота; паратиреомониторинг. 